\title{
EFEITO DA DISPONIBILIDADE DE SÍTIOS DE NIDIFICAÇÃO SOBRE O SISTEMA DE ACASALAMENTO E O CUIDADO PATERNAL EM UM OPILIÃO NEOTROPICAL
} (ARACHNIDA: OPILIONES)

Dissertação apresentada ao Instituto de Biociências da Universidade de São Paulo como parte dos requisitos para obtenção do título de Mestre em Ciências na área "Ecologia".

Orientador: Glauco Machado

Departamento de Ecologia, Universidade de São Paulo, Brasil 


\section{FICHA CATALOGRÁFICA}

FICHA CATALOGRÁFICA ELABORADA PELO SERVIÇO DE BIBLIOTECA DO INSTITUTO DE BIOCIÊNCIAS / USPP

W491 Werneck, Rachel Miranda

Efeito da disponibilidade de sítios de nidificação sobre o sistema de acasalamento e o cuidado paternal em um Opilião Neotropical (ARACHNIDA: OPILIONES) / Rachel Miranda Werneck -- São Paulo: R. M. Werneck, 2012.

36p. : il.

Dissertação (Mestrado) - Universidade de São Paulo, Instituto de Biociências, Departamento de Ecologia, 2012.

1. Canibalismo filial 2. Competição introssexual 3. Efeito de residência 4. Poligamia - Defesa de recursos 5. Seleção Sexual I. Universidade de São Paulo, Instituto de Biociências, Departamento de Ecologia. II. Título.

LC: QL 751

\section{COMISSÃO JULGADORA:}

$\operatorname{Prof}(a) \cdot \operatorname{Dr}(a)$.

$\operatorname{Prof}(a) . \operatorname{Dr}(a)$.

Prof(a). Dr.(a). 


\section{AGRADECIMENTOS}

Agradeço ao meu orientador, Glauco Machado, por me dar uma grande oportunidade, por sempre me questionar e criticar, fazendo com que eu aprendesse muito durante esse processo. Por toda a atenção e dedicação. Enfim, profissionalmente eu devo minhas conquistas à sua orientação.

À CAPES e ao Programa de Pós-graduação em Ecologia da Universidade de São Paulo, em particular, seus funcionários (Dalva, Bernadete, Maurício, Socorro, Vera, PC, Luís e Érika) e professores. Ao Donizete da Escola de Comunicação e Artes.

Aos membros da banca, que aceitaram o convite de participar da defesa e seguramente contribuirão para a melhoria deste trabalho.

Às minhas famílias, Mirandas, Wernecks e Contrins, especialmente aos avós paternos e maternos.

A todos os professores e colegas dos laboratórios, LAGE e Opilio Lab. Em especial, ao Gustavo Requena (Billy) pela co-orientação, ao Daniel Caetano (Musgo) e a Camila Zatz pela colaboração, ao Danilo pela companhia no bandejão e no café, ao Rodolfo pelas conversations e a Cristiane Millan pela amizade.

\section{Paulicéia desvairada}

Nesta seção, eu agradeço a todas as pessoas que me fizeram perder a vontade de ir embora e que são hoje a minha família.

\footnotetext{
"So no one told you life was gonna be this way

Your job is a joke, you're broke, your love life's D.O.A.

It's like you're always stuck in second gear

When it hasn't been your day, your week, your month, or even your year, but...

I'll be there for you, 'cause you're there for me too"
}

Maria Vianna, por compartilhar comigo um lar, a aventura de chegar em Sampa, as tristezas, a saudade, o vinho, a cerveja e também muitos momentos felizes! Denise Alves, por me apresentar um mundo melhor em Sampa, por ser, além de amiga, uma excelente profissional com quem eu pude contar em importantes etapas da minha vida acadêmica. Julia Beneti, por me mostrar que é possível encarar os fatos com mais leveza. Por ser uma amiga para todas as horas, para atividades gastronômicas, para cantar no carro, para fazer nada. Por cuidar dos meninos e por muitas outras coisas às quais eu serei eternamente grata. 
Eduardo Pinto, pelas infindáveis discussões, pelos conselhos, pelo carinho e por sempre alegrar nossas reuniões. Jomar Batista, por compartilhar os regionalismos e por sempre coordenar a festa. Camila Mandai, por aleatoriamente se tornar uma grande amiga, monitora particular do R e guia turística. Pela sua adorável e divertida forma de se expressar. Taís Nazareth, por ter chegado na hora certa, por me mostrar que as decepções serão superadas, por me ajudar sempre, por ser amiga, conselheira e ser minha referência bibliográfica. Marco Senna, por ser odiavelmente um poço de cultura do qual eu me aproveito, por ser muito companheiro nas horas de filmes e cafés e por ser um amigo muito querido. Paulo Machado, por ser um irmãozinho, companheiro de filmes, nerd poser head banger sweet friend. Julia Astegiano, por ser indelicadamente amiga, grosseiramente divertida e lerdamente querida. Porque os argentinos são mucho mas engraçados! Alejandra Tapia, por compartilhar comigo o lar doce lar, por seu uma tia dedicada, pela paciência e pelos ensinamentos em estatística e língua espanhola. Esther Sebastian, por sempre me encorajar, pelos conselhos e por estar sempre sorrindo. Laura Rocha Prado, por basicamente salvar a minha vida. Por ser uma irmã. Barbara Henning e Paula Condé, pelos 25 dias de treinamento intensivo para entender como é ter irmãs. Vocês conseguiram. E agora vão ter que me amar pra sempre! Tiago Faria, nunca fui sua amiga, nunca tive consideração por você, mas é assim, escolhi você pra ser meu irmão. Amor incondicional. Obrigada por sempre me ouvir, por sempre me xingar e por me apoiar. Aos amigos do IB: Mariana T., Sérgio, Pri, Natália Nery, Mitsuo, Jonathan, Mateus, Loboda e Ro, Pedro dos grilos, Flavinha, Ivy, Marcel, João, Sarah e Tat Kawamoto.

Aos amigos 'virtuais' que me acompanharam nesses quatro anos, comentando minhas reclamações, compartilhando o fardo de ser um pós-graduando, acompanhando o Opilio Night/Fight Club e me deixando falar (e falar muito) sobre o meu trabalho. Um agradecimento especial para o Fernando Castro, porque além de todos os fatos citados acima ele ainda pediu para ler essa dissertação! Ao Carlos Borges (OGeleia) por ser meu ombro amigo, conselheiro e pela sua delicadeza para jogar as verdades na minha cara. Ao Filipe Braga, um verdadeiro amigo que existe em letrinhas. Ao Marcelo P. Florido, meu melhor amigo imaginário, por dividir comigo as histórias dos biólogos de índios, por ser meu ombro amigo, conselheiro, destruidor da felicidade alheia (realista) e ainda assim, ser bizarramente adorável.

Ao Robert Smith, George Lucas, Douglas Adams, Marck Zuckerberg, Roger Waters, Tim Burton, Woody Allen, David Shore, Chuck Lorre, Carlos Drummond, Mario Quintana, Pablo Neruda e muitos outros... 


\section{ÍNDICE}

Resumo

Abstract

Introdução

\section{Métodos}

Coleta de dados em campo

Manutenção dos indivíduos em cativeiro

Descrição geral do experimento

Competição intrassexual

Custos do cuidado paternal

Procedimentos estatísticos gerais

Resultados

Observações em campo

Experimento de laboratório: dados descritivos

Experimento de laboratório: competição intrassexual

Experimento de laboratório: custos do cuidado paternal

Discussão

Referências 


\section{Resumo}

Cavidades naturais constituem um recurso reprodutivo monopolizável, cuja disponibilidade determina a intensidade da competição macho-macho que, por sua vez, pode influenciar os custos do cuidado paternal, pois quanto maior o risco de perda do recurso, menor deve ser a freqüência de forrageio dos machos. Fêmeas do opilião Magnispina neptunus utilizam cavidades naturais como sítios de nidificação, que são monopolizados por machos em um sistema de poliginia por defesa de recursos. Após a oviposição, as fêmeas abandonam os ninhos e todo o cuidado parental é exercido pelos machos. O objetivo deste trabalho foi avaliar o efeito da disponibilidade de ninhos sobre a competição intrassexual e os custos do cuidado paternal em M. neptunus. Dois grupos experimentais foram estabelecidos em laboratório: alta (8 ninhos) e baixa (4 ninhos) disponibilidade de sítios reprodutivos. Cada grupo era composto por sete terrários contendo 12 machos e 12 fêmeas. Apesar da redução dos sítios de nidificação ter reduzido o número de machos detentores de ninhos, o tamanho dos indivíduos não exerceu nenhum efeito sobre o sucesso de monopolização de ninhos. Aparentemente, o pequeno diâmetro de entrada dos ninhos experimentais reduz a importância do tamanho dos machos sobre sua probabilidade de monopolizar um sítio de nidificação. Apenas a residência prévia parece determinar o resultado das disputas entre machos e, portanto, os custos de abandonar os ninhos devem ser altos. De fato, machos guardiões permanecem quase todo o tempo dentro dos seus ninhos em ambos os grupos experimentais, o que aumenta os custos do cuidado. Como conseqüência, o canibalismo filial é freqüente, pois os custos em termos de redução no tamanho da desova são menores do que os benefícios de manter a posse do ninho e dos ovos, que sabidamente aumentam a atratividade dos machos e suas chances de obterem novas desovas.

Palavras-chave: canibalismo filial, competição intrassexual, efeito de residência, poliginia por defesa de recursos, seleção sexual. 


\section{Abstract}

Natural cavities are a reproductive resource that can be monopolized, and their availability determines the intensity of male-male competition, which in turn may influence the costs of paternal care since the higher the risk of loosing the resource, the low should be male foraging frequency. Females of the harvestman Magnispina neptunus use natural cavities as nest site, which are monopolized by males in typical resource defense polygyny mating system. After oviposition, females leave the nests and all parental activities are in charge of males. The goal of this study was to evaluate the effect of nest site availability on the intrasexual competition and the costs of paternal care in M. neptunus. Two experimental groups were established in the laboratory: high (8 nests) and low (4 nests) nest site availability. Each group was composed of seven terraria containing 12 males and 12 females. Although the reduction of in the number of nests sites has indeed reduced the number of males holding nests, male size did not influence the probability of acquiring and maintaining a nest. Apparently, the small diameter of entrance in the experimental nests reduces the importance of male size on their resource holding power. Only previous residence seems to influence the output of agonistic interactions between two males and, therefore, the costs of leaving the nest to forage are likely to be high. In fact, guarding males remain almost all the time inside their nests, which increases the costs of paternal care. As a consequence, filial cannibalism is frequent in both experimental groups because the costs in terms of reduction in clutch size are likely to lower than the benefits of holding a nest containing eggs, which is known to increase male attractiveness and his chance of acquiring additional eggs.

Key-words: filial cannibalism, intrasexual competition, resident effect, resource defense polygyny, sexual selection. 


\section{Introdução}

O termo sistema de acasalamento é amplamente usado para descrever as formas pelas quais sociedades animais estão estruturadas em relação ao comportamento sexual (Andersson, 1994). Um dos principais fatores que influencia o tipo de sistema de acasalamento exibido pelos indivíduos de uma população é a disponibilidade de recursos, principalmente alimento e sítios de reprodução (Emlen \& Oring, 1977). A distribuição e a disponibilidade desses recursos necessários para a reprodução determinam a distribuição espacial das fêmeas no ambiente, resultando em um potencial ambiental para a poliginia (PAP). Assim, se os recursos estiverem distribuídos de forma agregada, as fêmeas estarão em maior densidade no entorno dos recursos e haverá um alto PAP (Emlen \& Oring, 1977). A distribuição espacial e temporal das fêmeas, por sua vez, influencia a distribuição do número de cópulas entre machos. Machos que tiverem maior habilidade em monopolizar os recursos necessários para a reprodução (ou as fêmeas que se agregam em torno destes recursos) conseguirão um maior número de cópulas do que machos com menor habilidade. Portanto, a combinação entre o PAP e a habilidade de monopolização dos recursos pelos machos irão determinar a distribuição das cópulas e, consequentemente, o sistema de acasalamento na população e a intensidade da seleção sexual (Emlen \& Oring, 1977; Shuster \& Wade, 2003).

O monopólio do acesso aos recursos ou às parceiras reprodutivas por um macho impede ou dificulta que outros machos tenham acesso a cópulas. O grau de monopolização das cópulas pelos machos, entretanto, depende do quão defensáveis são os recursos ou as parceiras reprodutivas (Emlen \& Oring, 1977). Em um sistema de acasalamento baseado na defesa de fêmeas, o sucesso reprodutivo dos machos e a intensidade da competição intrassexual são predominantemente influenciados pela capacidade dos machos monopolizarem parceiras. Já em um sistema de acasalamento baseado na defesa de recursos, o sucesso reprodutivo dos machos e a intensidade da competição intrassexual são influenciados tanto pela capacidade dos machos monopolizarem os recursos quanto pela 
disponibilidade dos recursos (Ostfeld, 1987). Como a capacidade de manutenção de recursos (resource holding power ou RHP) dos indivíduos varia dentro de uma população, é esperado que machos com maior RHP obtenham um maior número de cópulas do que machos com menor RHP (revisão em Kelly, 2008). Adicionalmente, a variação no RHP aumenta a variação no sucesso reprodutivo entre os machos da população (Parker, 1974; Shuster \& Wade, 2003).

Outro componente importante dos sistemas de acasalamento é o cuidado parental, que pode influenciar as taxas reprodutivas potenciais e, consequentemente, a razão sexual operacional da população (Emlen \& Oring, 1977; Clutton-Brock \& Vincent, 1991). Da perspectiva masculina, um maior investimento na prole pode levar a uma redução na taxa reprodutiva potencial e a uma razão sexual operacional enviesada para fêmeas (e.g., Rosenqvist, 1990; Vincent et al., 1994; Smith, 1997). Entretanto, a maioria dos casos de cuidado paternal exclusivo em artrópodes, peixes e anfíbios ocorrem em linhagens com sistema de acasalamento poligínico, nas quais os machos usualmente defendem territórios ou sítios reprodutivos (Gross \& Shine, 1981; Zeh \& Smith, 1985; Ah-King et al., 2005; Wells, 2007). Nesses grupos, cuidar da prole e adquirir novas cópulas não são atividades mutuamente excludentes, ao contrário do que acontece com aves (revisão em Magrath \& Komdeur, 2003). Adicionalmente, o cuidado paternal pode prover um sinal honesto sobre a qualidade do macho como defensor da prole, de forma que indivíduos que cuidam da prole podem ser preferidos pelas fêmeas e, consequentemente, obterem um número maior de cópulas (Tallamy, 2000, 2001). De fato, evidências empíricas para peixes (e.g. Hoelzer, 1989; Lindström et al., 2006) e algumas espécies de artrópodes (e.g. Gilbert et al., 2010; Nazareth \& Machado, 2010) apóiam a predição de que fêmeas preferem machos com ovos.

O cuidado paternal, entretanto, pode impor custos energéticos quando o abandono da prole para forrageio resulta em redução da viabilidade da prole (Clutton-Brock, 1991). Machos do opilião Neotropical Iporangaia pustulosa, por exemplo, exibem um marcado 
decréscimo da condição corporal ao longo do período de cuidado a prole, pois os machos guardiões abandonam a prole com uma frequência relativamente baixa e, quando o fazem, raramente conseguem obter alimento (Requena et al., 2012). Uma forma de compensar esses custos do cuidado sem abandonar a prole é o canibalismo filial, definido como o consumo da própria prole pelo indivíduo parental (FitzGerald, 1992). De fato, o canibalismo filial é bastante comum quando há cuidado paternal e é mais provável ocorrer quando o consumo da prole pode aumentar a qualidade do cuidado (Manica, 2002, 2004; Thomas \& Manica, 2003; Klug \& Bonsall, 2007).

Cavidades naturais constituem um recurso reprodutivo monopolizável para vários organismos nidificadores secundários, ou seja, aqueles que utilizam cavidades pré-existentes no solo, em troncos, rochas ou mesmo conchas como sítio reprodutivo (e.g., peixes: Forsgreen et al., 1996; anuros: Heying, 2004; aves: Jacot et al., 2009). A disponibilidade das cavidades naturais determina a intensidade da competição intraespecífica por restringir a probabilidade de reprodução e, consequentemente, o sucesso reprodutivo dos indivíduos (Newton, 1994; Heinsohn, 2008). Se as cavidades naturais forem um recurso escasso, a competição entre os indivíduos deve ser intensificada e, portanto, a variável ambiental 'disponibilidade de ninhos' terá forte influência na intensidade da competição intrassexual. Nesse sentido, machos que possuem a maior capacidade de monopolizar as cavidades disponíveis no ambiente serão favorecidos e terão maior sucesso reprodutivo que machos que não tiveram acesso aos sítios reprodutivos (e.g., Slagsvold, 1986; Lindström, 1988; Borg et al., 2002). Portanto, em sistemas de acasalamento baseados na posse de sítios de nidificação escassos, é comum que a seleção sexual favoreça características morfológicas e comportamentais nos machos que aumentem seu RHP, tais como armamentos e habilidade de briga (e.g., Côte \& Hunte, 1989; Jamieson, 1995; Lindstrom \& Pampoulie, 2005). 
Magnispina neptunus (Gonyleptidae) é um opilião cujos indivíduos possuem atividade predominantemente noturna e utilizam cavidades naturais em barrancos como sítios de oviposição. A biologia reprodutiva de M. neptunus foi investigada por Nazareth \& Machado (2010), que caracterizaram o sistema de acasalamento da população estudada como uma poliginia por defesa de recursos, na qual os machos ocupam e defendem cavidades que servirão de ninho para as fêmeas deixarem seus ovos. As cópulas só ocorrem no interior dos ninhos e os machos cortejam as fêmeas antes, durante e após a cópula, até que terminem a oviposição. Após a oviposição, as fêmeas abandonam os ninhos e os ovos ficam sob o cuidado exclusivo dos machos. Como muitas fêmeas podem visitar o ninho de um mesmo macho, as desovas geralmente são formadas por ovos provenientes de cópulas múltiplas. Portanto, machos guardiões não têm seu sucesso reprodutivo limitado pelo cuidado paternal, mas sim pela posse de um ninho. Adicionalmente, a presença de ovos no ninho torna um macho mais atraente para as fêmeas, de forma que machos sem prole conseguem menos cópulas do que machos com ovos em seus ninhos. Entretanto, a freqüência com que os machos guardiões saem dos ninhos para forragear é menor do que a dos machos sem prole, o que deve comprometer sua condição física durante os quase dois meses de cuidado paternal. Apesar de machos guardiões forragearem com menor freqüência, nunca foram observados eventos de canibalismo filial em laboratório, onde a disponibilidade de ninhos foi sempre muito alta. Porém, tanto fêmeas quanto os machos entram em ninhos alheios e canibalizam ovos (Nazareth \& Machado, 2010).

Dada a importância de cavidades naturais para a biologia reprodutiva de $M$. neptunus, o objetivo deste trabalho foi testar experimentalmente o efeito da disponibilidade de sítios de nidificação sobre o sistema de acasalamento e os custos do cuidado paternal nesta espécie de opilião. Mais especificamente, serão testadas predições de duas hipóteses gerais: (1) a baixa disponibilidade de sítios de nidificação intensifica a competição entre machos que, por sua vez, (2) aumenta os custos associados ao cuidado paternal (Tabela 1). 
Tabela 1. Efeito da disponibilidade de sítios de nidificação (baixa vs. alta) sobre o sistema de acasalamento e os custos do cuidado paternal no opilião Magnispina neptunus.

\section{Predições}

\section{Baixa Alta}

\section{Competição intrassexual}

1.1. Número de mudanças de proprietário do ninho (takeovers)

maior menor

1.2. Efeito do tamanho do macho sobre a probabilidade de adquirir um ninho

maior menor

1.3. Efeito do tamanho do macho sobre a probabilidade de adquirir uma

maior menor desova

1.4. Probabilidade de um macho detentor de ninho conseguir uma desova

maior menor

\section{Custos do cuidado paternal}

2.1. Frequência com que os machos guardiões abandonam os ninhos para forragear

menor maior

2.2. Frequência de canibalismo filial

maior menor

2.3. Proporção de ovos canibalizados

maior menor

\section{Métodos}

\section{Coleta de dados em campo}

A população de $M$. neptunus utilizada neste trabalho ocorre em um fragmento de Mata Atlântica localizado em Arraial d'Ajuda (16²4'51.20"S; 3904'17.38"W), distrito de Porto Seguro, Bahia. Durante quatro dias, foram realizadas observações dos indivíduos em campo ao longo de duas parcelas retangulares estabelecidas em barrancos separados por um filete de água de cerca de $1 \mathrm{~m}$ de largura. Cada parcela tinha uma área de $17 \mathrm{~m}^{2}(17 \mathrm{~m}$ de 
comprimento $\times 1 \mathrm{~m}$ de altura) e as observações foram realizadas por duas pessoas, cada uma percorrendo uma das parcelas. Todos os ninhos encontrados nas duas parcelas foram localizados e marcados. Todos os machos com ninho assim como aqueles que se deslocavam dentro das parcelas foram medidos quanto à largura do escudo dorsal (uma medida de tamanho estrutural) e receberam uma marcação individual com tinta para aeromodelismo. Para os machos que tinham ninho, também foi medido o diâmetro da abertura dos ninhos. Todas as medidas foram tomadas utilizando um paquímetro digital com precisão de 0,01 $\mathrm{mm}$.

As variáveis tomadas em campo foram usadas para caracterizar o sistema de acasalamento de $M$. neptunus sob condições naturais e também para testar duas predições relacionadas à probabilidade de obtenção de um ninho e, subsequentemente, de uma desova. Esses dados foram usados mais tarde para comparar os resultados obtidos nos experimentos conduzidos em laboratório com aqueles obtidos em campo. De acordo com a primeira predição, o tamanho dos machos tem um efeito positivo sobre a probabilidade de adquirir um ninho. A segunda predição é de que o tamanho dos machos também tem um efeito positivo sobre a probabilidade de adquirir uma desova. Ambas as predições foram testadas pelo ajuste de modelos lineares generalizados com distribuição binomial de erros e função de ligação do tipo logit. Os modelos relacionam o tamanho dos machos (variável preditora contínua) com a probabilidade de adquirir: (a) um ninho (variável resposta binária com dois níveis: 0 = sem ninho, 1 = com ninho) ou (b) uma desova (variável resposta binária com dois níveis: $0=$ sem desova, $1=$ com desova). No caso da primeira predição, todos os machos encontrados em campo dentro das parcelas amostradas foram usados no teste. Para a segunda predição, somente os machos com ninho foram usados no teste, pois somente estes machos podem receber desovas (Nazareth \& Machado, 2010). 
Manutenção dos indivíduos em cativeiro

Em áreas adjacentes às parcelas descritas acima, foram coletados 168 machos e 168 fêmeas para o experimento em laboratório (veja descrição a seguir). Os indivíduos foram trazidos para o Instituto de Biociências da Universidade de São Paulo e acondicionados em terrários (54 x $27 \mathrm{~cm}$ de base, $23 \mathrm{~cm}$ de altura) contendo terra, folhas secas e fragmentos de cascas de árvores. Durante o período de estudo, as condições climáticas da sala de criação foram $25{ }^{\circ} \mathrm{C}$ de temperatura, $80 \%$ de umidade relativa do ar e fotoperíodo de 11:13 $\mathrm{h}$ (claro:escuro). Os indivíduos foram alimentados três vezes por semana com pedaços de larvas de tenébrio (Coleoptera: Tenebrionidae). Cada indivíduo recebeu uma marca individual de tinta no dorso e todos os machos tiveram a largura do escudo dorsal medida utilizando um paquímetro digital com precisão de $0,01 \mathrm{~mm}$.

\section{Descrição geral do experimento}

Foram montados 14 terrários, com 12 machos e 12 fêmeas em cada um deles. A seleção de cada conjunto de 12 machos levou em consideração o tamanho dos indivíduos de modo que a média e o desvio padrão da largura do escudo dorsal dos machos não diferiu entre os terrários $(6,67 \pm 0,59 \mathrm{~mm}$; ANOVA, $F=0,70, \mathrm{gl}=153, \mathrm{p}=0,76)$. Em seguida, os 14 terrários foram divididos equitativamente em dois grupos experimentais. No grupo experimental que simula alta disponibilidade de sítios de nidificação, cada terrário recebeu oito ninhos artificiais construídos com argila conforme protocolo descrito em Nazareth \& Machado (2010). Os ninhos artificiais possuíam as mesmas dimensões e a entrada de todos eles tinha $15 \mathrm{~mm}$ de diâmetro, que foi a média do diâmetro das entradas dos ninhos encontrados em campo $(15,11 \pm 5,77 \mathrm{~mm})$. Nos sete terrários restantes, foi simulada uma situação de baixa disponibilidade de sítios de nidificação, havendo quatro ninhos em cada terrário. De acordo com informações coletadas em campo, 45\% dos machos na população estudada possuíam um ninho. No caso do experimento realizado em laboratório, o grupo de 
alta disponibilidade de ninhos permitia que até $66,6 \%$ dos machos tivessem ninhos, enquanto esta porcentagem no grupo de baixa disponibilidade era de apenas 33,3\%.

Cada terrário foi acompanhado por 30 a 34 dias, durante os quais foi realizado o acompanhamento noturno da atividade dos indivíduos. A cada noite de observação foram realizadas seis a oito vistorias em cada ninho, com intervalos regulares de 30 min entre cada uma das vistorias. Em cada vistoria foi registrada a ocupação de cada um dos ninhos, a identidade dos ocupantes e o comportamento dos indivíduos dentro dos ninhos, incluindo cortejo, cópula, brigas e eventos de oviposição. Os ninhos com desovas foram fotografados após cada evento de oviposição para a quantificação do número de ovos depositado pelas fêmeas. Como só era possível acompanhar quatro terrários simultaneamente, o experimento foi realizado em quatro baterias entre os meses de julho e novembro de 2011. As três primeiras baterias incluíram dois terrários de baixa e dois de alta disponibilidade de ninhos em cada uma delas. A quarta bateria incluiu apenas um terrário de baixa e um de alta disponibilidade de ninhos. Os procedimentos específicos para o teste de cada uma das predições descritas na Tabela 1 são detalhados a seguir.

\section{Competição intrassexual}

Para testar a predição 1.1, sobre o número de mudanças de proprietário do ninho (Tabela 1), foram registradas todas as vezes em que um macho detentor de um ninho (dono) foi substituído por outro macho. Foram considerados donos somente os machos observados por, no mínimo, 18 vistorias consecutivas (i.e., três dias) dentro do mesmo ninho. A predição foi testada pelo ajuste de modelos lineares generalizados com efeitos mistos (GLMMs) com distribuição de erros Poisson. O modelo relaciona o número de vezes que houve substituição do dono de cada ninho nos dois grupos experimentais (alta e baixa disponibilidade de ninhos), levando em consideração a identidade de cada terrário como fator de agrupamento (variável aleatória). Esse modelo foi comparado ao modelo nulo, que simula um cenário no 
qual não há nenhum efeito dos grupos experimentais sobre o número de vezes que os ninhos mudam de dono.

Para testar a predições 1.2 e 1.3, sobre o efeito do tamanho do macho sobre a probabilidade de adquirir um ninho e uma desova, respectivamente (Tabela 1), foram utilizados procedimento similares. Em ambos os casos, o tamanho de cada macho foi estimado pela largura do escudo dorsal. A aquisição do ninho (variável resposta binária com dois níveis: $0=$ sem ninho, $1=$ com ninho) foi considerada apenas após o macho ter permanecido no ninho por mais de 18 vistorias consecutivas. Já a aquisição de desova (variável resposta binária com dois níveis: $0=$ sem desova, $1=$ com desova) foi considerada quando um macho obteve ovos em seu ninho. Ambas as predições foram testadas pelo ajuste de GLMMs com distribuição binomial de erros e função de ligação do tipo logit. Foram construídos quatro modelos que relacionam a probabilidade de adquirir um ninho ou uma desova com efeitos aditivos e de interação entre o tamanho dos machos e os grupos experimentais, tendo a identidade de cada terrário como variável aleatória. Ao contrário da predição 1.2, cuja análise considerou todos os machos, a análise predição 1.3 levou em consideração somente os machos que eram proprietários de ninhos, assim como foi feito com os dados obtidos em campo (veja tópico Coleta de dados em campo).

Finalmente, para testar a predição 1.4, sobre a probabilidade de um macho detentor de um ninho adquirir uma desova (Tabela 1), a variável resposta era composta por dois vetores: número de machos detentores de ninho com e sem desova. A predição foi testada pelo ajuste de GLMMs com distribuição binomial de erros. O modelo relaciona a proporção de machos detentores de ninho com e sem desova com a disponibilidade de ninhos (grupos experimentais), tendo a identidade de cada terrário como variável aleatória. 


\section{Custos do cuidado paternal}

Para testar a predição 2.1, sobre a freqüência de saídas dos machos guardiões (Tabela 1), foi considerado um evento de saída quando, em uma vistoria, o macho detentor de um ninho (i.e., aquele que já ocupava este ninho há, no mínimo, 18 vistorias consecutivas) não se encontrava dentro do seu ninho. Foi contabilizado também o número de vistorias nas quais esses machos estavam dentro de seus respectivos ninhos. A variável resposta, portanto, é do tipo binomial, composta por dois vetores: número de observações dentro e número de observações fora do ninho para cada um dos machos detentores de ninho. A predição foi testada pelo ajuste de GLMMs com distribuição de erros binomial. O modelo relaciona a freqüência com que os machos saem do ninho com a disponibilidade de ninhos (grupos experimentais), levando em consideração a identidade do terrário como variável aleatória.

Para testar a predição 2.2, sobre a freqüência de canibalismo filial (Tabela 1), foi considerado um evento de canibalismo filial quando, no momento da vistoria, o macho estava comendo ovos dentro do seu próprio ninho. As vistorias nas quais o macho estava dentro do ninho apenas cuidando da desova foram consideradas insucessos, havendo portanto uma variável resposta binomial composta por dois vetores: número de observações nas quais ocorreu canibalismo filial e o número de observações nas quais os machos estavam cuidando da prole sem praticar canibalismo filial. A predição foi testada pelo ajuste de GLMMs com distribuição de erros binomial. O modelo relaciona a freqüência com que os machos canibalizaram suas desovas com o efeito da disponibilidade de ninhos (grupos experimentais), levando em consideração a identidade do terrário como variável aleatória.

Por fim, para testar a predição 2.3, sobre a proporção de ovos canibalizados (Tabela 1), foi quantificada a redução no número de ovos em cada desova utilizando as fotografias sequenciais. Subtraindo o número total de ovos das fotos tomadas após o evento de canibalismo filial do número total de ovos presentes na desova em uma foto anterior ao evento de canibalismo, obteve-se o número de ovos canibalizados. Havia, portanto, uma 
variável resposta binomial composta por dois vetores: o número de ovos canibalizados e restante de ovos não canibalizados na desova. A predição foi testada pelo ajuste de GLMMs com distribuição de erros binomial. O modelo relaciona a proporção de ovos canibalizados por desova com o efeito da disponibilidade de ninhos (grupos experimentais), levando em consideração a identidade do terrário como variável aleatória.

\section{Procedimentos estatísticos gerais}

Os terrários foram considerados como variável aleatória em todos os casos para controlar fatores indesejados que podem gerar heterogeneidade entre eles, tal como a assincronia temporal na tomada de dados. Como as observações foram feitas em quatro baterias entre julho e novembro, os dados para cada terrário foram coletados em diferentes momentos da estação reprodutiva de $M$. neptunus. Além disso, para todos os modelos descritos acima, havia um modelo nulo concorrente que previa não haver nenhum efeito das variáveis preditoras sobre a variável resposta. A seleção dos modelos foi baseada nos valores de AICc, que consiste no critério de informação de Akaike (AIC) corrigido pelo tamanho amostral e é a forma recomendada para seleção modelos com menos de 40 unidades amostrais (Bolker et al., 2008; Symonds \& Moussalli, 2011). Usando comparativamente os valores de AICc, foram selecionados, dentre os modelos concorrentes, aqueles modelos que apresentaram o menor valor de $\triangle \mathrm{AICc}$. Os modelos com $\triangle \mathrm{AICc}$ menores do que 2 foram considerados igualmente plausíveis (Burnham \& Anderson, 2002). As análises foram realizadas no programa R ( $R$ Development Core Team, 2011) usando a função glmer do pacote lme4 (Bates, 2011) para a construção dos modelos e a função AICctab do pacote bblme (Bolker, 2011) para as comparações dos AICc entre modelos concorrentes. Todos os coeficientes dos modelos apresentados nos resultados referem-se ao grupo de baixa disponibilidade, de forma que valores positivos significam que a probabilidade de ocorrência de um determinado evento era maior neste grupo quando comparado ao grupo 
de alta disponibilidade. Da mesma forma, valores negativos significam que a probabilidade de ocorrência de um determinado evento era menor no grupo de baixa disponibilidade quando comparado ao grupo de alta.

\section{Resultados}

\section{Observações em campo}

Foram observados 109 machos de M. neptunus, sendo que 49 (45\%) destes tinham ninho. O tamanho médio $( \pm \mathrm{DP})$ dos machos detentores de ninhos foi $6,87 \pm 0,46 \mathrm{~mm}$, enquanto o tamanho médio dos machos que não conseguiram ninho foi $6,59 \pm 0,58 \mathrm{~mm}$. $\mathrm{O}$ modelo que melhor se ajustou aos dados foi aquele que considera o efeito do tamanho do macho sobre a probabilidade de adquirir um ninho (Tabela 2). Em concordância com a predição, machos maiores têm maior probabilidade de conseguir um ninho (coef. $=0,97$, erro $=0,38, \mathrm{p}=0,009 ;$ Figura 1$)$.

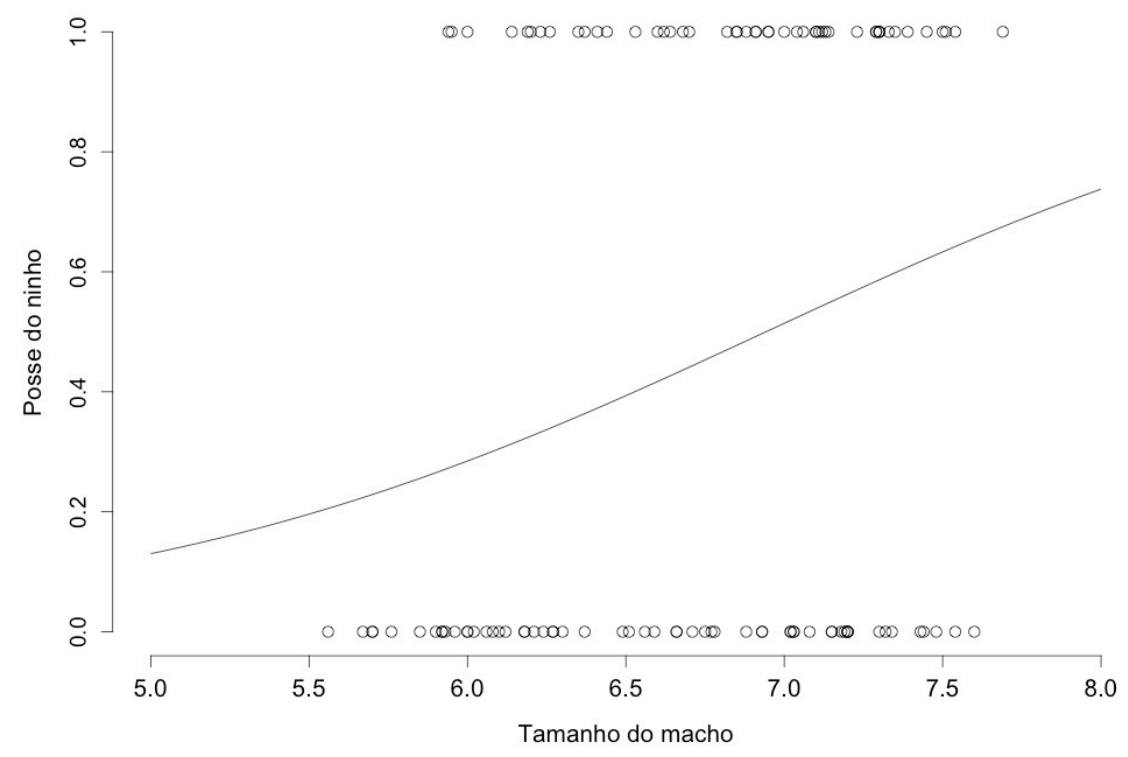

Figura 1. Efeito do tamanho dos machos de Magnispina neptunus sobre a probabilidade de possuir (1) ou não (0) um ninho em campo. A curva representa o ajuste do melhor modelo (ver Tabela 2). 
Dentre os 49 machos com ninho, $25(51 \%)$ tinham desova. O tamanho médio $( \pm D P)$ dos machos que possuíam desova foi $6,76 \pm 0,43 \mathrm{~mm}$, enquanto os machos que não possuíam

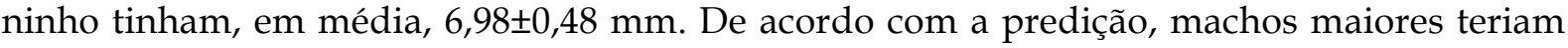
maior probabilidade de conseguir desovas. No entanto, o modelo que melhor se ajustou aos dados foi o modelo nulo, no qual se assume que não há efeito do tamanho dos machos sobre a probabilidade de adquirir uma desova (Tabela 2).

\section{Experimento de laboratório: dados descritivos}

Antes de apresentar os resultados dos testes das predições listadas na Tabela 1, serão apresentados aqui alguns dados descritivos gerais que serão usados para uma caracterização mais completa do sistema de estudo. Na condição de baixa disponibilidade, seis dos sete terrários tiveram todos os quatro ninhos ocupados. A porcentagem máxima de machos detentores de ninho em cada terrário poderia chegar a $33 \%$ e a média obtida foi de $29 \%$. Na condição de alta disponibilidade, nenhum dos terrários teve todos os oito ninhos ocupados. A porcentagem máxima de machos detentores de ninho em cada terrário deste grupo experimental, em tese, poderia chegar a $66 \%$, porém a média obtida foi de $44 \%$. Apesar da capacidade máxima de ocupação não ter sido alcançada, a porcentagem de machos com ninhos na condição de alta disponibilidade foi, em média, 15\% superior à condição de baixa disponibilidade. Esses valores sugerem que a redução experimental dos sítios de nidificação pode, de fato, ter intensificado a competição intrassexual.

Na condição de baixa disponibilidade, o número de machos com desova por terrário variou entre zero e quatro. Em cada terrário, uma média $( \pm D P)$ de 2,14 $\pm 1,34$ machos obtiveram desovas. Na condição de alta disponibilidade, o número de machos com desovas por terrário variou entre dois e sete, sendo a média igual a $2,85 \pm 1,34$. Na condição de baixa disponibilidade, a média do número de ovos por desova foi de $228,5 \pm 146,5$ enquanto na condição de alta disponibilidade, a média foi de $285,8 \pm 188,1$. Finalmente, foram observadas 
16 brigas entre machos pela posse de ninhos, sendo 10 delas na condição de baixa disponibilidade e seis na condição de alta disponibilidade. Nesses casos, os machos detentores de ninhos nunca perderam a disputa e os intrusos sempre foram repelidos.

\section{Experimento de laboratório: competição intrassexual}

Foram registradas apenas 15 trocas de proprietário nos 14 terrários ao longo de todo o período de duração do experimento. Nove trocas ocorreram em seis ninhos do grupo de baixa disponibilidade e sete trocas ocorreram em seis ninhos do grupo de alta disponibilidade. O modelo que melhor se ajustou aos dados foi o nulo, no qual não há efeito da disponibilidade de ninhos sobre a troca de proprietários (Tabela 3). O tamanho dos machos que conseguiram usurpar ninhos foi, em média, apenas $4 \%$ maior do que os machos que ocupavam previamente esses ninhos, porém existe uma enorme variação nos dados e $34 \%$ dos machos usurpadores eram menores que os ocupantes prévios (Figura 2).

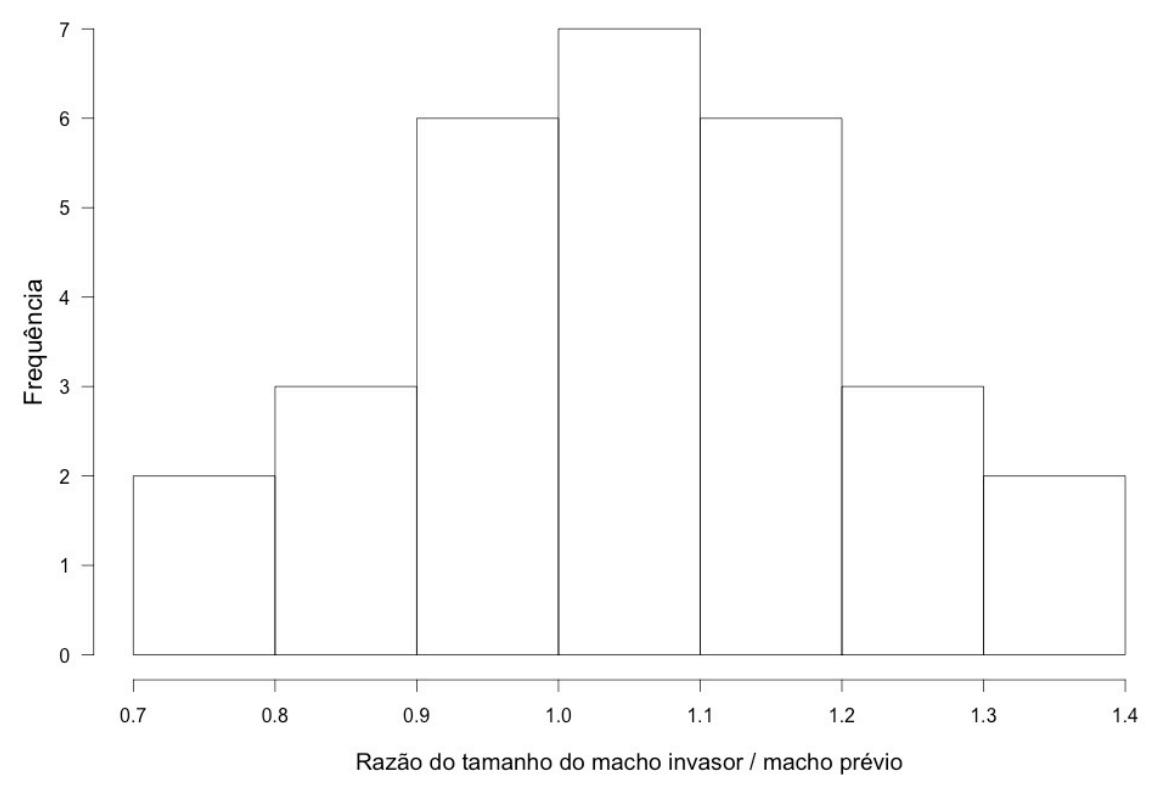

Figura 2. Histograma com a razão entre o tamanho do macho invasor e o tamanho do macho que previamente detinha um ninho. Tanto machos na condição de baixa quanto na de alta disponibilidade de ninhos foram incluídos aqui. 
Tabela 2. Tabela com os valores de AICc (Critério de Informação de Akaike ) para os modelos que avaliam o efeito do tamanho dos machos de Magnispina neptunus sobre a probabilidade de adquirir ninhos ou desovas tanto em campo quanto em laboratório. $\mathrm{k}=$ número de parâmetros no modelo; $\triangle \mathrm{AICc}=$ diferença entre o modelo em questão e o modelo com melhor ajuste aos dados; peso = verossimilhança relativa do modelo; $\mathrm{N}=$ probabilidade de adquirir um ninho $(0=$ sem ninho, $1=$ com ninho); $\mathrm{T}=$ tamanho do macho (largura do escudo dorsal); $\mathrm{D}=$ probabilidade de adquirir uma desova ( 0 = sem desova, 1 = com desova); $\mathrm{t}=$ identidade do terrário usada como fator aleatório; GE = grupo experimental que simula duas condições de disponibilidade de ninhos (alta ou baixa); * = interação entre as variáveis. Em todos os casos, o modelo com melhor ajuste está marcado em negrito.

\begin{tabular}{|c|c|c|c|c|c|}
\hline & Modelo & AICc & $\mathbf{k}$ & $\overline{\Delta A I C c}$ & Peso \\
\hline \multicolumn{6}{|l|}{ Campo } \\
\hline Efeito to tamanho do macho sobre & $\mathbf{N} \sim \mathbf{T}$ & 147,0 & 2 & 0,0 & 0,9262 \\
\hline a probabilidade de obter ninho & $\mathrm{N} \sim 1$ & 152,0 & 1 & 5,1 & 0,0738 \\
\hline Efeito to tamanho do macho sobre & $\mathbf{D} \sim \mathrm{T}$ & 69,4 & 2 & 0,0 & 0,569 \\
\hline a probabilidade de obter desova & $\mathrm{D} \sim 1$ & 70,0 & 1 & 0,6 & 0,431 \\
\hline \multicolumn{6}{|l|}{ Laboratório } \\
\hline \multirow{5}{*}{$\begin{array}{l}\text { Efeito to tamanho do macho sobre } \\
\text { a probabilidade de obter ninho } \\
\text { (predição 1.2) }\end{array}$} & $\mathbf{N} \sim \mathrm{G}+\mathrm{t}$ & 224,2 & 3 & 0,0 & 0,4362 \\
\hline & $\mathrm{N} \sim 1+\mathrm{t}$ & 225,4 & 2 & 1,2 & 0,2349 \\
\hline & $\mathrm{N} \sim \mathrm{T}+\mathrm{GE}+\mathrm{t}$ & 226,1 & 4 & 1,9 & 0,1706 \\
\hline & $\mathrm{N} \sim \mathrm{T}+\mathrm{t}$ & 227,3 & 3 & 3,1 & 0,0906 \\
\hline & $\mathrm{N} \sim \mathrm{T}+\mathrm{GE}+\mathrm{T}^{*} \mathrm{GE}+\mathrm{t}$ & 227,9 & 5 & 3,7 & 0,0677 \\
\hline \multirow{5}{*}{$\begin{array}{l}\text { Efeito to tamanho do macho sobre } \\
\text { a probabilidade de obter desova } \\
\text { (predição 1.3) }\end{array}$} & $D \sim 1+t$ & 89,0 & 2 & 0,0 & 0,5100 \\
\hline & $D \sim G E+t$ & 90,9 & 3 & 1,8 & 0,2031 \\
\hline & $\mathrm{D} \sim \mathrm{T}+\mathrm{t}$ & 91,1 & 3 & 2,1 & 0,1777 \\
\hline & $\mathrm{D} \sim \mathrm{T}+\mathrm{GE}+\mathrm{t}$ & 93,1 & 4 & 4,1 & 0,0672 \\
\hline & $\mathrm{D} \sim \mathrm{T}+\mathrm{GE}+\mathrm{T}^{*} \mathrm{GE}+\mathrm{t}$ & 94,0 & 5 & 5,0 & 0,0420 \\
\hline
\end{tabular}


O tamanho médio $( \pm \mathrm{DP})$ dos machos detentores de ninhos na condição de baixa disponibilidade foi de 6,75 0 ,55 $\mathrm{mm}$ enquanto na condição de alta disponibilidade, o tamanho médio dos machos foi de $6,65 \pm 0,58 \mathrm{~mm}$. O modelo com melhor ajuste aos dados foi aquele que considera apenas o efeito da disponibilidade de ninhos sobre a probabilidade de obter um ninho (coef. $=-0,58$, erro $=0,32, p=0,07$, Tabela 2 ), sem levar em conta o tamanho dos machos. De acordo com esse modelo, uma menor quantidade de ninhos diminui a probabilidade de uma macho ser detentor de um ninho, assim como seria esperado caso a manipulação experimental, de fato, intensificasse a competição intrassexual. Entretanto, como o valor de $\triangle \mathrm{AICc}$ entre esse modelo e o modelo nulo é menor do que 2 , ambos são igualmente plausíveis (Tabela 2).

O tamanho médio $( \pm \mathrm{DP})$ dos machos que obtiveram desova na condição de baixa disponibilidade foi de $6,85 \pm 0,6 \mathrm{~mm}$, enquanto na condição de alta disponibilidade o tamanho médio dos machos foi de $6,6 \pm 0,48 \mathrm{~mm}$. O modelo que melhor se ajustou aos dados foi o modelo nulo. O segundo melhor modelo, tão plausível quanto o nulo, inclui o efeito da disponibilidade de ninhos sobre a probabilidade de aquisição de desovas (coef. $=0,44$, erro $=$ $0,71, \mathrm{p}=0,53$, Tabela 2). Dentre os machos detentores de ninho, a probabilidade de conseguir uma desova não foi influenciada pela disponibilidade de ninhos. $\mathrm{O}$ modelo que melhor se ajustou aos dados foi o modelo nulo, porém o modelo que considera o efeito do grupo experimental foi igualmente plausível (coef. $=0,32$, erro $=0,73, p=0,65$, Tabela 3).

\section{Experimento de laboratório: custos do cuidado paternal}

Na condição de baixa disponibilidade de ninhos, em 11 dos 16 ninhos com desova houve saída do macho guardião para forragear. Em média $( \pm D P)$, os machos guardiões

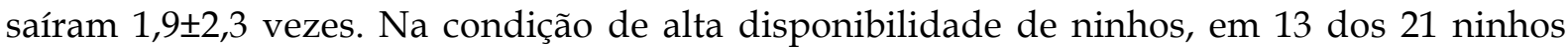
com desova os machos guardiões saíram para forragear. O número médio $( \pm D P)$ de saídas para cada macho foi de 1,38 $\pm 1,53$. O modelo que melhor se ajustou aos dados foi o modelo 
nulo, que assume não haver efeito da disponibilidade de ninhos sobre a frequência de saída dos machos guardiões (Tabela 3).

Tabela 3. Tabela com os valores de AICc (Critério de Informação de Akaike) para os modelos que avaliam o efeito da disponibilidade de ninhos sobre o sistema de acasalamento e o cuidado paternal em Magnispina neptunus. $\mathrm{k}=$ número de parâmetros no modelo; $\quad$ AICc $=$ diferença entre o modelo em questão e o modelo com melhor ajuste aos dados; peso = verossimilhança relativa do modelo; $\mathrm{t}$ = identidade do terrário usada como fator aleatório; $\mathrm{M}$ = número de mudanças de proprietário do ninho; $\mathrm{GE}=$ grupo experimental que simula duas condições de disponibilidade de ninhos (alta ou baixa); $\mathrm{D}=$ probabilidade de um macho com ninho adquirir uma desova $(0=$ sem desova, $1=$ com desova $) ; \mathrm{FA}=$ número de vezes que os machos abandonam os ninhos; $\mathrm{CF}=$ eventos de canibalismo filial; $\mathrm{C}=$ proporção de ovos canibalizados. Em todos os casos, o modelo com melhor ajuste está marcado em negrito.

\begin{tabular}{|c|c|c|c|c|c|}
\hline & Modelo & $\overline{A I C c}$ & $\mathbf{k}$ & $\mathrm{AICc}$ & Peso \\
\hline \multicolumn{6}{|l|}{ 1. Competição intrassexual } \\
\hline Número de mudanças de proprietário & $\mathbf{M} \sim \mathrm{GE}+\mathrm{t}$ & 74,0 & 3 & 0,0 & 0,603 \\
\hline do ninho (predição 1.1) & $\mathrm{M} \sim 1+\mathrm{t}$ & 74,8 & 2 & 0,8 & 0,397 \\
\hline Probabilidade de um macho conseguir & $D \sim 1+t$ & 88,3 & 2 & 0,0 & 0,733 \\
\hline uma desova (predição 1.4) & $\mathrm{D} \sim \mathrm{GE}+\mathrm{t}$ & 90,3 & 3 & 2,0 & 0,267 \\
\hline \multicolumn{6}{|l|}{ 2. Custos do cuidado paternal } \\
\hline Frequência de abandono de ninhos com & $\mathrm{FA} \sim \mathbf{1 + t}$ & 64,3 & 2 & 0,0 & 0,687 \\
\hline por machos guardiões (predição 2.1) & $\mathrm{FA} \sim \mathrm{GE}+\mathrm{t}$ & 65,8 & 3 & 1,6 & 0,313 \\
\hline Frequência de canibalismo filial & $\mathrm{CF} \sim 1+\mathrm{t}$ & 36,1 & 2 & 0,0 & 0,682 \\
\hline (predição 2.2) & $\mathrm{CF} \sim \mathrm{GE}+\mathrm{t}$ & 37,6 & 4 & 1,5 & 0,318 \\
\hline Proporção de ovos canibalizados & $C \sim 1+t$ & 575,7 & 2 & 0,0 & 0,554 \\
\hline (predição 2.3) & $\mathrm{C} \sim \mathrm{GE}+\mathrm{t}$ & 576,2 & 3 & 0,4 & 0,446 \\
\hline
\end{tabular}


Por fim, na condição de baixa disponibilidade de ninhos, ocorreram cinco eventos de canibalismo filial e foram canibalizados, em média $( \pm D P), 11,4 \pm 27,5$ ovos. De um total de 3.199 ovos em todos os terrários desse grupo experimental, 159 (5\%) foram consumidos pelos machos guardiões. Na condição de alta disponibilidade de ninhos, ocorreram sete eventos de canibalismo filial e foram canibalizados, em média $( \pm \mathrm{DP}), 16,1 \pm 22,4$ ovos. De um total de 4.572 ovos em todos os terrários desse grupo experimental, 257 (5,6\%) foram consumidos pelos machos guardiões. O modelo com melhor ajuste aos dados foi o modelo nulo, que assume não haver efeito da disponibilidade de ninhos sobre a proporção de ovos canibalizados (Tabela 3).

\section{Discussão}

Os dados obtidos em campo para Magnispina neptunus indicam que cavidades naturais são um recurso escasso, pois menos de $50 \%$ dos machos na população estudada eram detentores de um ninho. Levando em conta que a probabilidade de captura e detecção de machos não territoriais (i.e., sem ninho) deve ser consideravelmente mais baixa que a de machos territoriais (e.g., Buzatto et al., 2011), é possível que a porcentagem de machos sem acesso a cavidades naturais seja ainda maior na natureza. Portanto, a competição intrassexual pelo acesso e pela posse do recurso reprodutivo deve ser intensa e somente os machos de melhor qualidade, ou seja, aqueles com maior RHP, devem ser capazes de obter um ninho. De fato, a largura do escudo dorsal dos machos influenciou positivamente a probabilidade de um macho obter um ninho em campo (Figura 1). Em opiliões, assim como em muitos outros artrópodes, o tamanho dos armamentos masculinos é fortemente influenciado pelo tamanho corporal, de forma que indivíduos maiores possuem maior probabilidade de obterem e manterem a posse de territórios ou recursos reprodutivos (Emlen, 2008; veja exemplos com opiliões em Buzatto \& Machado, 2008; Zatz et al., 2011). 
Após adquirir um ninho, um macho é visitado por uma ou mais fêmeas que, em teoria, poderiam usar o tamanho do detentor do ninho como indicador de sua qualidade genética ou da sua habilidade como defensor da prole (Tallamy, 2001; Alonzo, 2012). Portanto, machos maiores deveriam ter uma maior probabilidade de adquirir desova, o que não foi encontrado em campo. De fato, em uma meta-análise a respeito do efeito do RHP sobre o sucesso reprodutivo dos machos em vários grupos animais, ficou demonstrado que a correlação entre essas duas variáveis é relativamente baixa (Kelly, 2008). Aparentemente, fêmeas de M. neptunus selecionam parceiros utilizando outras características sinalizadoras de qualidade que não estão intrinsecamente relacionadas ao tamanho dos machos. Características do sítio de nidificação, como sua dimensão e umidade interna, podem influenciar a probabilidade de sobrevivência dos ovos e, consequentemente, a decisão das fêmeas em ovipor ou não (veja exemplo com opiliões em Osses et al., 2008). As fêmeas também podem avaliar outras características do macho, como a intensidade e a qualidade do cortejo, que não necessariamente estão correlacionadas com o tamanho do macho (e.g., Tallamy et al., 2002).

A relação entre RHP e a probabilidade de obter um ninho observada em campo não foi encontrada no experimento realizado em laboratório, tanto na condição de alta quanto na de baixa disponibilidade de sítios de nidificação. Uma hipótese para explicar a ausência de padrão encontrada no laboratório baseia-se na defensabilidade do recurso que, no caso de $M$. neptuns, deve ser fortemente influenciada pela abertura de entrada do ninho. Ao contrário do que ocorre em campo, o diâmetro de abertura de todos os ninhos usados no experimento era idêntico (15 mm). Quanto menor a abertura do ninho, mais defensável ele deve ser e menor a importância do tamanho do macho sobre o resultado de interações agonísticas pela posse do ninho. Um padrão similar já foi descrito para a abelha Osmia lignaria propinqua (Megachilidae), cujas fêmeas defendem cavidades usadas como sítio de nidificação (Tepedino \& Torchio, 1994). Quando o diâmetro de abertura é grande, espera-se que o 
tamanho dos machos tenha uma importância maior no resultado das interações agonísticas de forma que indivíduos maiores devem ter vantagens sobre os menores. Os dados obtidos em campo são congruentes com essa hipótese dado que, nas cavidades naturais com diâmetro menor que $15 \mathrm{~mm}$, há uma grande variação no tamanho dos machos e tanto machos grandes quanto pequenos foram encontrados com ninhos (Figura 3). Em cavidades naturais com diâmetro superior a $15 \mathrm{~mm}$, entretanto, a variação no tamanho dos machos com ninho foi consideravelmente menor e $84 \%$ deles possuíam tamanho consideravelmente superior à média (Figura 3).

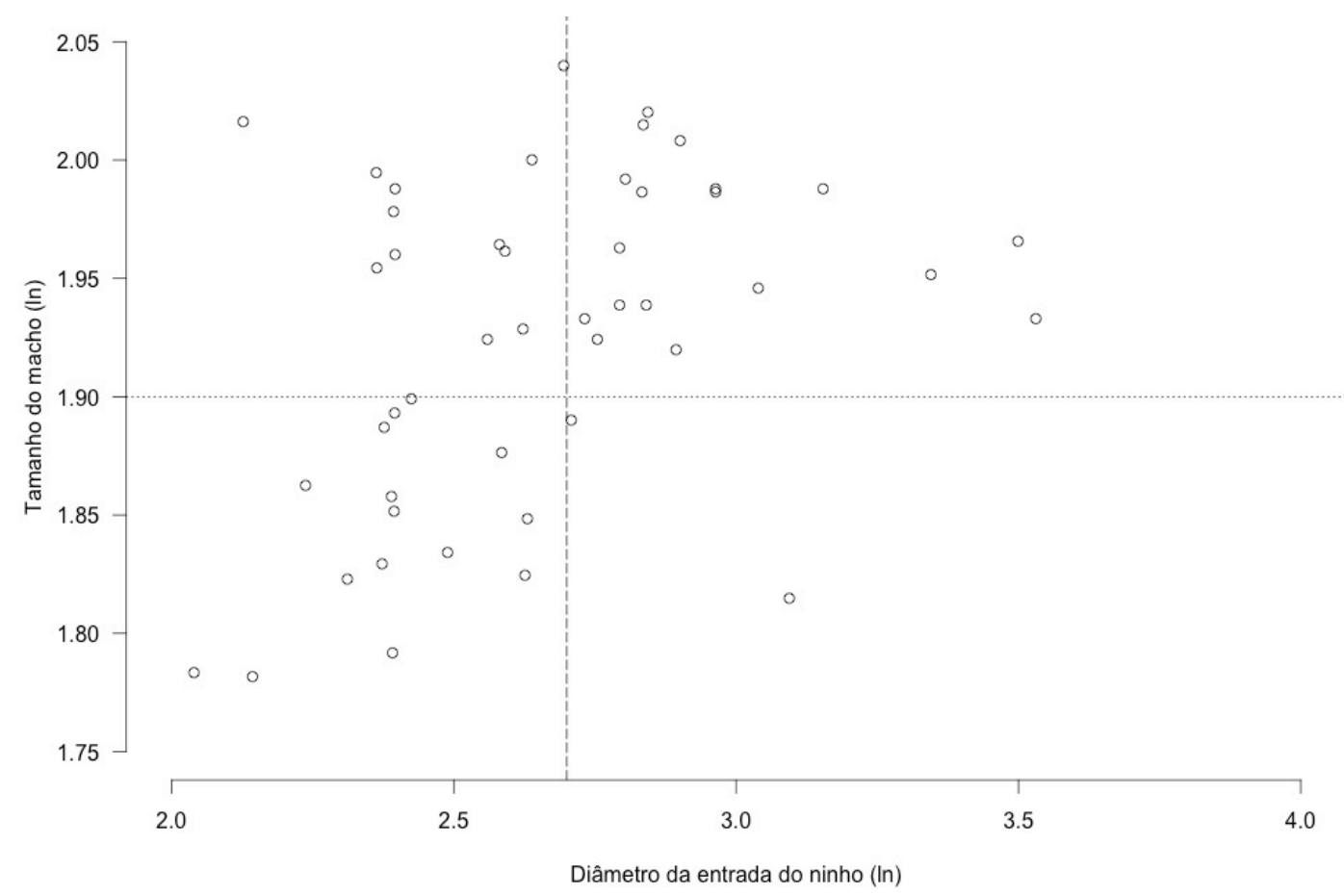

Figura 3. Correlação entre o diâmetro de entrada dos ninhos e o tamanho dos machos detentores desses ninhos em uma população do opilião Magnispina neptunus. A linha tracejada vertical corresponde a um ninho com diâmetro de entrada igual a $15 \mathrm{~mm}$, similar àquele que foi usado no experimento de laboratório. A linha pontilhada horizontal corresponde à média de tamanho dos machos detentores de ninhos em campo. Os dados foram logaritmizados para que o padrão fosse mais facilmente visualizado. 
A hipótese da defensabilidade do recurso apresentada aqui também pode explicar porque o número de mudanças de proprietários registrado no experimento foi tão baixo e não foi influenciado pela disponibilidade de sítios de nidificação. Nesse caso, a residência prévia, mais do que a diferença de tamanho entre os machos, deve ser o principal determinante do resultado das interações agonísticas entre os machos, assim como ocorre em várias espécies de insetos que defendem territórios (veja Kemp, 2000). Um dos mecanismos subjacentes ao efeito de residência em $M$. neptunus pode ser a vantagem de estar dentro do ninho e poder bloquear sua entrada usando o quarto par de pernas dotado de fortes espinhos. De fato, em um estudo prévio realizado com a espécie em laboratório, machos guardiões foram capazes de bloquear a entrada de seus ninhos impedindo a entrada de coespecíficos (Machado \& Nazareth, 2010). Fêmeas da abelha Osmia lignaria propinqua também defendem seus ninhos estando com o corpo inteiramente dentro deles e, independentemente do seu tamanho, são capazes de repelir a maioria das intrusas (Tepedino \& Torchio, 1994)

Um outro mecanismo subjacente ao efeito de residência em M. neptunus, que deve se aplicar somente a condições naturais, é a manobrabilidade. Se cavidades com entradas pequenas possuem um espaço interno exíguo, machos menores podem ter vantagens em interações agonísticas com machos maiores. Em besouros do gênero Onthophagus (Scarabaeidae), machos defendem túneis construídos sob o esterco e seus chifres exercem um papel importante na defesa do recurso, pois bloqueiam a entrada do túnel e impedem o acesso de rivais (veja Emlen \& Philips, 2006). Entretanto, já foi demonstrado que machos com chifres pequenos, apesar de não se engajarem em combates, manobram melhor dentro dos túneis estreitos (Moczek \& Emlen, 2000; Madewell \& Moczek, 2006). Se o mesmo ocorre com M. neptunus, machos menores podem ser mais eficientes do que machos grandes em repelir invasores de dentro de ninhos pequenos. Novamente, esta hipótese é congruente com os 
dados obtidos em campo, pois 59\% dos machos detentores de ninhos pequenos (com diâmetro de entrada inferior a $15 \mathrm{~mm}$ ) possuíam tamanho inferior à média (Figura 3).

Apesar do número de machos detentores de ninho ter sido menor na condição de baixa disponibilidade de sítios de nidificação, a maior competição intrassexual não aumentou a probabilidade de um macho adquirir desova quando comparada aos machos na condição de maior disponibilidade. Infelizmente, não existem informações detalhadas sobre os critérios de seleção feminina em $M$. neptunus, o que dificulta a interpretação dos resultados obtidos em laboratório. O que se sabe é que, a partir do momento em que um macho adquire uma primeira desova, sua atratividade aumenta muito em comparação a machos com ninho sem desovas (Nazareth \& Machado, 2010). Portanto, o valor associado ao sítio de nidificação também aumenta e os machos deveriam investir ainda mais em sua defesa (Arnott \& Elwood, 2008; Hollander et al., 2012). De fato, nenhuma mudança na identidade dos machos detentores de ninhos foi observada após um determinado macho adquirir uma desova.

Se machos residentes possuem uma grande vantagem frente a machos rivais em interações agonísticas (independentemente da diferença de tamanho entre eles), os custos de abandonar um ninho contendo ovos são muito elevados em ambas as condições experimentais de disponibilidade de sítios de nidificação. Portanto, não é surpreendente que a freqüência com que os machos guardiões abandonaram os ninhos para forragear tenha sido muito baixa e similar nos dois grupos experimentais. Dado que não foi encontrada diferença na freqüência de forrageio dos machos guardiões nos dois grupos experimentais, não seria esperado encontrar diferenças na freqüência de canibalismo filial e na proporção de ovos canibalizados. De fato, tanto na condição de alta quanto na de baixa disponibilidade de ninhos, machos guardiões consumiram ovos de suas desovas em frequência e quantidade similares. Diante da supressão quase total do forrageio, o canibalismo filial pode minimizar os custos energéticos do cuidado paternal sem o risco de perder a posse do sítio de 
nidificação e dos ovos nele contidos (Manica, 2002, 2004). Resultados similares foram obtidos para o percevejo predador Rhinocoris tristis, cujos machos cessam o forrageio durante a guarda da prole e, durante todo o período de cuidado paternal, alimentam-se exclusivamente de ovos de suas próprias desovas (Thomas \& Manica, 2003). Apesar de não haver um ninho, machos rivais estão constantemente tentando usurpar desovas, pois isso aumenta muito a atratividade dos machos (Gilbert et al., 2010). Portanto, da mesma forma que em M. neptunus, os custos de abandonar os ovos são muito altos em $R$. tristis, o que pode explicar a alta freqüência de canibalismo filial nesta espécie de percevejo.

Em um trabalho prévio no qual as condições de criação eram muito similares às do experimento conduzido neste estudo, porém quase todos os machos de M. neptunus possuíam ninhos, a freqüência de forrageio dos machos guardiões foi consideravelmente mais alta do que a registrada aqui e não foi registrado nenhum evento de canibalismo filial (Nazareth \& Machado, 2010). Como os indivíduos utilizados aqui e no estudo prévio provêm de localidades diferentes e isoladas, é possível que a ocorrência de canibalismo filial seja decorrente de diferenças comportamentais entre os indivíduos das duas populações. Uma outra possibilidade plausível é que, em uma situação na qual os custos de abandonar os ninhos são relativamente baixos, os benefícios do canibalismo filial não compensem os custos em termos de diminuição do sucesso reprodutivo dos machos (Manica, 2004). Portanto, em condições naturais, seria esperado que machos detentores de ninhos com entrada pequena fossem mais propensos ao canibalismo filial porque os riscos de abandonar a desova e perder definitivamente a posse do sítio e dos ovos nele contidos seriam maiores quando comparados a machos detentores de ninhos com entrada grande.

Em conclusão, apesar da redução experimental dos sítios de nidificação ter intensificado a competição intrassexual em M. neptunus, reduzindo o número de machos detentores de ninhos, o tamanho dos machos não exerceu nenhum efeito sobre o sucesso de obtenção e manutenção de sítios de nidificação. Aparentemente, características intrínsecas 
dos ninhos experimentais, como o pequeno diâmetro de suas entradas, têm um efeito importante reduzindo a importância do RHP sobre a probabilidade de um macho ser detentor de um sítio de nidificação. Como conseqüência, a residência prévia parece ter um papel importante para determinar o resultado das disputas entre machos e os custos de abandonar os ninhos devem ser muito altos. Portanto, machos guardiões permanecem quase todo o tempo dentro dos seus ninhos, o que aumenta muito os custos do cuidado. Nesse cenário, o canibalismo filial deve ser favorecido, pois os custos em termos de redução no tamanho da desova devem ser superados pelos benefícios de manter a posse do ninho e dos ovos, que sabidamente aumentam a atratividade dos machos e suas chances de obterem novas desovas.

\section{Referências}

Ah-King, M., Kvarnemo, C. \& Tullberg, B. S. 2005. The influence of territoriality and mating system on the evolution of male care: a phylogenetic study on fish. Journal of Evolutionary Biology 18: 371-382

Alonzo, S. H. 2012. Sexual selections favours male parental care, when females can choose. Proceedings of the Royal Society B 279: 1784-1790

Andersson, M. 1994. Sexual selection. Princeton University Press, Princeton

Arnott, G. \& Elwood, R. W. 2008. Information gathering and decision making about resource value in animal contests. Animal Behaviour 76: 529-542

Bates, D., Maechler, M. \& Bolker, B. 2011. lme4: Linear mixed-effects models using S4 classes. R package 618 version 0.999375-42. http:/ /CRAN.R-project.org/package=lme4

Bolker, B. M. 2011. bbmle: Tools for general maximum likelihood estimation. R package 616 version 1.0.3. http://CRAN.R-project.org/package=bbmle 
Bolker, B. M., Brooks M. E., Clark C. J., Geange, S. W., Poulsen J. R., Stevens M. H. H. \& White, J. S. 2008. Generalized linear mixed models: a practical guide for ecology and evolution. Trends in Ecology and Evolution 24: 127-135

Borg, A. A., Forsgren, E. \& Magnhagen, C. 2002. Plastic sex-roles in the common goby - the effect of nest availability. Oikos 98: 105-115

Burnham, K. P. \& Anderson, D. R. 2002. Model selection and multimodel inference. Springer, New York

Buzatto, B. A. \& Machado, G. 2008. Resource defense polygyny shifts to female defense polygyny over the course of the reproductive season of a Neotropical harvestman. Behavioral Ecology and Sociobiology 63: 85-94

Buzatto, B. A., Requena, G. S., Lourenço, R. S., Munguía-Steyer, R. \& Machado, G. 2011. Conditional male dimorphism and alternative reproductive tactics in a Neotropical arachnid (Opiliones). Evolutionary Ecology 25: 331-349

Clutton-Brock, T. H. 1991. The evolution of parental care. Princeton University Press, Princeton

Clutton-Brock, T. H. \& Vincent, A. C. J. 1991. Sexual selection and the potential reproductive rates of males and females. Nature 351: 58-60

Côte, I. M. \& Hunte, W. 1989. Male and female mate choice in the redlip blenny: why bigger is better. Animal Behaviour 38: 78-88

Emlen, D. J. 2008. The evolution of animal weapons. Annual Review of Ecolology, Evolution and Systematics 39: 387-413

Emlen, D. J. \& Philips, K. T. 2006. Phylogenetic evidence for an association between tunneling behavior and the evolution of horns in dung beetles (Coleoptera: Scarabaeidae: Scarabaeinae). Coleopterists Society Monograph 5: 47-56

Emlen, S. T. \& Oring, L. W. 1977. Ecology, sexual selection, and the evolution of mating systems. Science 197:215-223 
FitzGerald, G. J. 1992. Filial cannibalism in fishes: why do parents eat their offspring? Trends in Ecology and Evolution 7: 07-10

Forsgren E., Kvarnemo, C. \& Lindström K. 1996. Mode of sexual selection determined by resource abundance in two sand goby populations. Evolution 50: 646-654

Gilbert, J. D. J., Thomas, L. K. \& Manica, A. 2010. Quantifying the benefits and costs of parental care in assassin bugs. Ecological Entomology 35: 639-651

Gross, M. R. \& Shine, R. 1981. Parental care and mode of fertilization in ectothermic vertebrates. Evolution 35: 775-793

Heinsohn, R. 2008 The ecological basis of unusual sex roles in reverse-dichromatic electus parrots. Animal Behaviour 76: 97-103

Heying, H. 2004. Reproductive limitation by oviposition site in a treehole breeding Madagascan poison frog (Mantella laevigata). Miscellaneous Publications of the Museum of Zoology 193: 23-30

Hoelzer, G. A. 1989. Male-male competition and female choice in the Cortez damselfish, Stegastes rectifraenum. Animal Behaviour 40: 339-349

Hollander, F. A., Titeux, N. \& Dyck, H. V. 2012. Territorial resource valuation deviates from habitat quality in an ecologically trapped, long-distance migratory bird. Behavioral Ecology and Sociobiology 66: 777-783

Jacot, A., Valcu, M., van Oers, K., \& Kempenaers, B. 2009 Experimental nest site limitations affects reproductive strategies and parental investment in a hole-nesting passerine. Animal Behaviour 77: 1075-1083

Jamieson, I. 1995. Do female fish prefer to spawn in nests with eggs for reasons of mate choice copying or egg survival? American Naturalist 145: 824-832

Kelly, C. D. 2008. The interrelationships between resource-holding potential, resource-value and reproductive success in territorial males: how much variation can we explained? Behavioral Ecology and Sociobiology 62: 855-871 
Kemp, D. J. 2000. Contest behavior in territorial male butterflies: does size matter? Behavioral Ecology 11: 591-596

Klug, H. \& Bonsall, M. B. 2007 When to care for, abandon, or eat your offspring: the evolution of parental care and filial cannibalism. American Naturalist 170: 886-901

Lindström, K. 1988. Male-male competition for nest sites in the sand goby, Pomatoschistus minutus. Oikos 53: 67-73

Lindström, K. \& Pampoulie, C. 2005. Effects of resource holding potential and resource value on tenure at nest sites in sand gobies. Behavioral Ecology 16: 70-74

Lindström, K., St. Mary, C. M. \& Pampoulie, C. 2006. Sexual selection for male parental care in the sand goby, Pomatoschistus minutus. Behavioral Ecology and Sociobiology 60: 46-51

Madewell, R. \& Moczek, A. P. 2006. Horn possession reduces maneuverability in the hornpolyphenic beetle, Onthophagus nigriventis. Journal of Insect Science 6: 10, available online: insectscience.org/6.21

Magrath, M. J. L. \& Komdeur, J. 2003. Is male care compromised by additional mating opportunity? Trends in Ecology and Evolution 18: 424-430

Manica, A. 2002. Filial cannibalism in teleost fish. Biological Reviews 77: 261-277

Manica, A. 2004. Parental fish change their cannibalistic behaviour in response to the cost-tobenefit ratio of parental care. Animal Behaviour 67: 1015-1021

Moczek, A. P. \& Emlen, D. J. 2000. Male horn dimorphism in the scarab beetle, Onthophagus taurus: do alternative reproductive tactics favour alternative phenotypes? Animal Behaviour 59: 459-466

Nazareth, T. M. \& Machado, G. 2010. Mating system and exclusive postzygotic paternal care in a Neotropical harvestman (Arachnida: Opiliones). Animal Behaviour 79:547-554

Newton, I. 1994. The role of nest sites in limiting the numbers of hole-nesting birds: a review. Biological Conservation 70: 265-276 
Osses F., Martins, E. G. \& Machado, G. 2008 Oviposition site selection by the neotropical bromeliad-dweller harvestman Bourguyia hamata (Arachnida: Opiliones). Journal of Ethology 26: 233-241

Ostfeld, R. S. 1987. On the distinction between female defense and resource defense polygyny. Oikos 48:238-240

Parker, G. A. 1974. Assessment strategy and the evolution of fighting behavior. Journal of Theoretical Biology 47:223-243

R Development Core Team. 2011. R: A language and environment for statistical computing. R Foundation for Statistical Computing, Vienna, Austria. ISBN 3-900051-07-0, URL http:/ / www.R-project.org

Requena, G. S., Buzatto, B. A., Martins, E. G. \& Machado, G. 2012. Paternal care decreases foraging activity and body condition, but does not impose survival costs to caring males in a Neotropical arachnid. PLos One no prelo

Rosenqvist, G. 1990. Male mate choice and female-female competition for mates in the pipefish Nerophis ophidion. Animal Behaviour 39: 1110-1115

Shuster, S. M. \& Wade, M. J. 2003. Mating systems and strategies. Princeton University Press, Princeton

Slagsvold, T. 1986. Nest site settlement by the pied flycatcher: does the female choose her mate for the quality of his house or himself? Ornis Scandinavica 17: 210-220

Smith, R. L. 1997. Evolution of paternal care in the giant water bugs (Heteroptera: Belostomatidae), pp. 116-149. J.C. Choe \& B.J. Crespi (Eds.), The evolution of social behavior in insects and arachnids. Cambridge University Press, Cambridge

Symonds, M. R. E. \& Moussalli, A. 2011. A brief guide to model selection, multimodel inference and model averaging in behavioural ecology using Akaike's information criterion. Behavior Ecology and Sociobiology 65: 13-21 
Tallamy, D. W. 2000. Sexual selection and evolution of exclusive paternal care in arthropods. Animal Behaviour 60: 559-567

Tallamy, D. W. 2001. Evolution of exclusive paternal care in arthropods. Annual Review of Entomology 46:139-165

Tallamy, D. W., Powell, B. E. \& McClafferty, J. A. 2002. Male traits under cryptic female choice in the spotted cucumber beetle (Coleoptera: Chrysomelidae). Behavioral Ecology 13: $511-518$

Tepedino, V. J. \& Torchio, P. F. 1994. Founding the usurping equally efficient paths to nesting success in Osmia lignaria propinqua (Hymenoptera, Megachilidae). Annals of the Entomological Society of America 87:946-953

Thomas, L. K. \& Manica, A. 2003. Filial cannibalism in an assassin bug. Animal Behaviour 66: 205-210

Vincent, A., Ahnesjö, I. \& Berlung, A. 1994. Operational sex ratios and behavioural sex differences in a pipefish population. Behavioral Ecology and Sociobiology 34: 142-135

Wells, D. K. 2007. The ecology and behavior of amphibians. University of Chicago Press, Chicago

Zatz, C., Werneck, R. M., Macías-Ordóñez, R. \& Machado, G. 2011. Alternative mating tactics in dimorphic males of the harvestman Longiperna concolor (Arachnida: Opiliones). Behavioral Ecology and Sociobiology 65: 995-1005

Zeh, D. W. \& Smith, R. L. 1985 Paternal investment by terrestrial arthropods. Integrative and Comparative Biology 25: 785-805 